\title{
Chemical coupling of thiolated chitosan to preformed liposomes improves mucoadhesive properties
}

This article was published in the following Dove Press journal:

International Journal of Nanomedicine

18 May 2012

Number of times this article has been viewed

\author{
Kerstin Gradauer' \\ Caroline Vonach' \\ Gerd Leitinger ${ }^{2,3}$ \\ Dagmar Kolb 2,3 \\ Eleonore Fröhlich ${ }^{3}$ \\ Eva Roblegg ${ }^{4}$ \\ Andreas Bernkop-Schnürch ${ }^{5}$ \\ Ruth Prass| ${ }^{1,6}$ \\ 'Institute of Biophysics and \\ Nanosystems Research, Austrian \\ Academy of Sciences, Graz, Austria; \\ 'Institute of Cell Biology, Histology, \\ and Embryology, Medical University \\ of Graz, Graz, Austria; ${ }^{3}$ Center \\ for Medical Research, Medical \\ University of Graz, Graz, Austria; \\ ${ }^{4}$ Institute of Pharmaceutical Sciences/ \\ Pharmaceutical Technology, Karl- \\ Franzens University, Graz, Austria; \\ ${ }^{5}$ Department of Pharmaceutical \\ Technology, Institute of Pharmacy, \\ University of Innsbruck, Innsbruck, \\ Austria; ${ }^{\circ}$ Ludwig Boltzmann Institute \\ for Lung Vascular Research, Graz, \\ Austria
}

Aim: To develop mucoadhesive liposomes by anchoring the polymer chitosan-thioglycolic acid (chitosan-TGA) to the liposomal surface to target intestinal mucosal membranes.

Methods: Liposomes consisting of phosphatidylcholine (POPC) and a maleimide-functionalized lipid were incubated with chitosan-TGA, leading to the formation of a thioether bond between free SH-groups of the polymer and maleimide groups of the liposome. Uncoated and newly generated thiomer-coated liposomes were characterized according to their size, zeta potential, and morphology using photon correlation spectroscopy and transmission electron microscopy. The release behavior of calcitonin and the fluorophore/quencher-couple ANTS/DPX (8-aminonaphthalene-1,3,6-trisulfonic acid/p-xylene-bis- pyridinium bromide) from coated and uncoated liposomes, was investigated over 24 hours in simulated gastric and intestinal fluids. To test the mucoadhesive properties of thiomer-coated and uncoated liposomes in-vitro, we used freshly excised porcine small intestine.

Results: Liposomes showed a concentration-dependent increase in size - from approximately $167 \mathrm{~nm}$ for uncoated liposomes to $439 \mathrm{~nm}$ for the highest thiomer concentration used in this study. Likewise, their zeta potentials gradually increased from about $-38 \mathrm{mV}$ to $+20 \mathrm{mV}$, clearly indicating an effective coupling of chitosan-TGA to the surface of liposomes. As a result of mucoadhesion tests, we found an almost two-fold increase in the mucoadhesion of coupled liposomes relative to uncoupled ones. With fluorescence microscopy, we saw a tight adherence of coated particles to the intestinal mucus.

Conclusion: Taken together, our current results indicate that thiomer-coated liposomes possess a high potential to be used as an oral drug-delivery system.

Keywords: thiomer, liposome, mucoadhesion, chitosan-thioglycolic acid, oral drug delivery

\section{Introduction}

The oral route of drug delivery is considered the most convenient route for patients and medics alike, since it allows for easy, painless administration, thus leading to a high patient compliance. Nevertheless, several drugs, particularly peptide and protein drugs, still have to be administered intravenously due to enzymatic degradation and/or low absorption in the gastrointestinal tract. Different delivery systems have been developed to enhance the oral bioavailability of such drugs, among them liposomes. Liposomes are lipid nanoparticles, which have already shown their potential as drug carriers to deliver sensitive drugs to specific targets. ${ }^{1-3}$ Although liposomes showed some problems with reproducibility when they were first developed as oral drug carriers, they seem to be promising delivery systems with a good biocompatibility and a high versatility. ${ }^{4,5}$ To improve the properties of liposomes for oral delivery, various
Correspondence: Ruth Prassl

Institute of Biophysics and Nanosystems Research, Austrian Academy of Sciences Schmiedlstraße 6A-8042,

Graz-Messendorf,Austria

Tel +433164120305

Fax +433164120390

Email ruth.prass!@oeaw.ac.at 
strategies have been designed, such as combining liposomes with permeation enhancers, or coating them with polymers like chitosan, carbopol, eudragit, or silica. ${ }^{6-11}$ Polymers used for this coating procedure provide an interesting characteristic for oral drug delivery: they interact with mucosal surfaces, thereby increasing the time the delivery system is trapped at the site of drug absorption. Longer et al first showed that a delayed gastrointestinal transit, as induced by bioadhesive polymers, has a high potential of increasing the oral bioavailability of drugs. ${ }^{12}$ Hence, mucoadhesive delivery systems have been developed not only for oral but also for buccal, rectal, pulmonary, ocular, or nasal drug delivery. ${ }^{13-15}$

In the present study, we aimed to develop a novel delivery system for the oral application of drugs, based on the coating of liposomes with a specific class of polymers called thiomers. Thiomers are prepared by immobilizing agents with thiol groups, such as L-cysteine, thioglycolic acid, 6-mercaptonicotinic acid, or 2-imminothiolane $\mathrm{HCl}$, on polymeric backbones like polycarbophil, chitosan, or sodium carboxymethylcellulose. ${ }^{16-21}$ Their main advantage is the increase in mucoadhesion relative to unmodified polymers. Unmodified polymers interact with mucin layers via non-covalent bonds, such as ionic interactions or hydrogen bonds, whereas thiomers are thought to form disulfide bridges with cysteine-rich subdomains of mucus glycoproteins. ${ }^{16}$ Furthermore, thiomers have been shown to act as permeation enhancers by temporarily opening tight junctions in a reversible manner, which could further enhance the uptake of drugs. ${ }^{22}$

The objective of this study was to investigate the possibility of taking chitosan-thioglycolic acid (chitosan-TGA) as a coating material for lipid nanoparticles. Chitosan has been chosen because of its biocompatibility, biodegradability, and safe toxicity profile. ${ }^{23}$ In contrast to a loose coupling of chitosan to the liposomal surface, which is mostly achieved by ionic interactions between polymer and liposomes, we covalently anchored the thiolated chitosan to the liposome. ${ }^{24-26}$ We then investigated the morphology of the newly generated particles, as well as the influence of the polymer coating on the size and zeta potential of the liposomes, their release behavior, and their mucoadhesive properties.

\section{Materials and methods Materials}

Palmitoyl-oleoyl-phosphatidylcholine (POPC); 1,2dioleoyl-sn-glycero-3-phosphoethanolamine-N-[4-(pmaleimidomethyl)cyclohexane-carboxamide] (DOPE-MCC); and 1,2-dioleoyl-sn-glycero-3-phosphoethanolamine-N(lissamine rhodamine B sulfonyl) were purchased from
Avanti Polar Lipids (Alabaster, AL). Chitosan-thioglycolic acid (chitosan-TGA) - with a molecular weight of $77 \mathrm{kDa}$ and $539 \pm 57 \mu \mathrm{mol} \mathrm{SH}$-groups/g polymer - was synthesized and provided by Thiomatrix (Innsbruck, Austria). Fluorescein isothiocyanat (FITC)-labeled calcitonin (32 amino acids; molecular weight: $3803.29 \mathrm{~g} / \mathrm{mol}$ ) was synthesized and provided by piCHEM (Graz, Austria). The phosphate buffer ( $\mathrm{pH}$ 7.4) used for mucoadhesion studies was purchased from Invitrogen (Darmstadt, Germany). All other chemicals were of reagent grade and purchased from Sigma-Aldrich (Vienna, Austria).

\section{Preparation of liposomes}

Stock solutions of POPC and DOPE-MCC were prepared in chloroform-methanol $(2: 1 \mathrm{v} / \mathrm{v})$ and chloroform, respectively. Aliquots of both stock solutions were mixed to obtain a molar ratio of POPC:DOPE-MCC of 3:0.3 for the coupling of chitosan-TGA. For control liposomes, pure POPC was used. To test mucoadhesive behavior, the fluorescent phospholipid 1,2dioleoyl-sn-glycero-3-phosphoethanolamine-N-(lissamine rhodamine B sulfonyl) was added to both phospholipid solutions, giving a final ratio of 3.3:0.01 (lipid to label). The organic solvent was evaporated under a stream of nitrogen to obtain a lipid film, which was dried overnight in a vacuum chamber to ensure complete removal of the organic solvent. Hydration of the dry lipid film was accomplished by adding $10 \mathrm{mM}$ phosphate buffer containing $150 \mathrm{mM} \mathrm{NaCl}, \mathrm{pH} 7.4$ (PBS), to yield a final lipid concentration of $30 \mathrm{mg} / \mathrm{mL}$. The lipid suspension was incubated for one hour at room temperature with repeated vortexing. The resulting multilamellar vesicles were sized by freeze and thaw (six cycles), followed by extrusion through $200 \mathrm{~nm}$ polycarbonate membranes (Whatman Inc, Clifton, NJ) with a Mini-Extruder (Avanti Polar Lipids, Alabaster, AL).

To investigate the release from coated and uncoated liposomes, the liposomes were loaded either with anionic fluorophore 8-aminonaphthalene-1,3,6-trisulfonic acid (ANTS) and the cationic quencher p-xylene-bis-pyridinium bromide (DPX), or with FITC-labeled calcitonin. Hydration of the POPC/DOPE-MCC film was performed with $5.4 \mathrm{mg} / \mathrm{mL}$ of ANTS and $19 \mathrm{mg} / \mathrm{mL}$ of DPX in PBS, or with $200 \mu \mathrm{g} / \mathrm{mL}$ of FITC-calcitonin in PBS, followed by freeze and thaw and size extrusion, as described.

\section{Coupling of chitosan-TGA}

Coupling was accomplished by the formation of thioether bonds between free SH-groups of chitosan-TGA and functionalized maleimide-groups of the liposome (see Figure 1). 


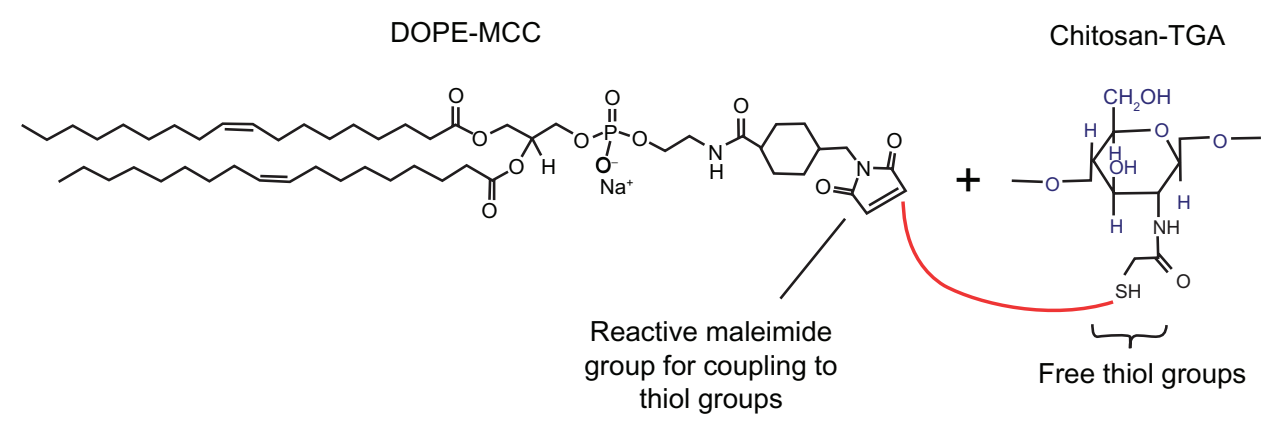

Figure I Reaction scheme for the covalent coupling of chitosan-TGA to a maleimide-functionalized phospholipid to form a stable thioether bond. Abbreviations: DOPE-MCC, I,2-dioleoyl-sn-glycero-3-phosphoethanolamine-N-[4-(p-maleimidomethyl)cyclohexane-carboxamide]; TGA, thioglycolic acid.

Chitosan-TGA was synthesized according to the method described previously. ${ }^{17}$ Different amounts of polymer, which were dissolved in deionized water to achieve a concentration of $3 \mathrm{mg} / \mathrm{mL}$, were added to liposomal suspensions, which were diluted via PBS to a concentration of $10 \mathrm{mg} / \mathrm{mL}$, to obtain molar ratios of $1: 6,1: 4,1: 2,1: 1,2: 1,4: 1$, and $6: 1$ (SH-groups:maleimide groups). The mixtures were incubated overnight under agitation at room temperature. Control liposomes were treated the same way.

\section{Ellman's test}

A photometric assay was performed to determine the amount of free SH-groups of chitosan-TGA according to a slightly modified version of the Ellman's method. ${ }^{27}$ Briefly, the Ellman's reagent 5,5'-dithiobis-(2-nitrobenzoic acid) (DTNB) was dissolved in water to a concentration of $7.5 \mathrm{mM}$ and the $\mathrm{pH}$ was adjusted by adding $\mathrm{NaHCO}_{3}$. L-cysteine was used as standard substance and a standard curve was prepared in a concentration range from 1 to $80 \mu \mathrm{M}$. Samples and standard substance were diluted to $800 \mu \mathrm{l}$ with phosphate buffer (100 mM Na-phosphate, 15 mM NaCl, 1 mM EDTA; pH 7.4) followed by the addition of $200 \mu \mathrm{LTNB}$-solution. The reaction was allowed to proceed for 30 minutes at room temperature in the dark. The amount of SH-groups was determined by measuring the absorbance at $412 \mathrm{~nm}$ using a Hitachi U-2000 spectrophotometer (Hitachi, Tokyo, Japan).

To determine the total amount of thiol groups after coupling, samples with molar ratios of 4:1 and 6:1 (SH-groups to maleimide groups, respectively) were used. In addition to coupled liposomes, control liposomes, mixed with the same amount of polymer, were also measured. All samples were reduced prior to the Ellman's test by adding $65 \mu \mathrm{L}$ of freshly prepared $1 \%$ sodium borohydride $\left(\mathrm{NaBH}_{4}\right)$ solution to $100 \mu \mathrm{L}$ of liposomal suspension. After incubation for 1 hour at room temperature under argon and agitation, excess $\mathrm{NaBH}_{4}$ was removed by adding the same amount of $1 \mathrm{M} \mathrm{HCl}$.
The Ellman's test was accomplished as described; however, before measuring, the turbid liposomes had to be removed. For this purpose, centrifugal filter units with a cutoff of $10 \mathrm{kDa}$ (Millipore, Vienna, Austria) were used (swinging bucket rotor at $5000 \mathrm{~g}$ for 20 minutes at $4^{\circ} \mathrm{C}$ ). The flow through (yellowcolored reaction product) was transferred to a plastic cuvette and the absorbance was measured immediately.

\section{Particle size determination}

Particle size distribution was determined by photon correlation spectroscopy using a Zetasizer 3000HSA (Malvern Instruments, Herrenberg, Germany), which operated with a $10 \mathrm{~mW}$ helium-neon laser at a wavelength of $632.8 \mathrm{~nm}$. The scattered light was measured at an angle of $90^{\circ}$ and the temperature was maintained at $25^{\circ} \mathrm{C}$. Particle size was analyzed by calculating the auto correlation function of the detected intensity; the polydispersity index of the liposomal suspension was given by the width of the size distribution. Coupled and control liposomes were measured after diluting them to a final lipid concentration of $0.03 \mathrm{mg} / \mathrm{mL}$ with ultrapure water (USF ELGA, High Wycombe, UK).

\section{Determination of the zeta potential}

The zeta potential of coupled and control liposomes was determined using the Zetasizer Nano ZS (Malvern Instruments) - according to the Helmholtz-Smoluchowski equation - from the mobility of the liposomes in an oscillating electric field. Samples were diluted with a buffer containing $10 \mathrm{mM}$ of Tris and $2 \mathrm{mM}$ of $\mathrm{CsCl}(\mathrm{pH} 7.0)$ to a lipid concentration of $0.3 \mathrm{mg} / \mathrm{mL}$, and were measured using a folded capillary cell (Malvern Instruments).

\section{Negative-staining transmission electron microscopy}

A total of $10 \mu \mathrm{L}$ of coated or uncoated POPC/DOPE-MCC liposomes (3 mg/mL) was placed on a carbon-over-Pioloform ${ }^{\circledR}$ - 
coated copper grid and incubated for 1 minute. The excess sample was blotted with filter paper and immediately replaced by $10 \mu \mathrm{L}$ of staining agent, which was allowed to settle for 2 minutes; then, it was blotted again. Ammoniummolybdate $(5 \%)$, phosphotungstic acid (1\%), and uranyl acetate $(2 \%)$ were tried as staining agents. Visualization of the samples was performed using a Zeiss EM 902 transmission electron microscope (Carl Zeiss Microscopy, Oberkochen, Germany) at an acceleration voltage of $80 \mathrm{kV}$. Digital images were made using a Proscan Slow Scan CCD camera at $1 \times 1 \mathrm{~K}$ resolution.

\section{Freeze fracture transmission electron microscopy}

Coated and uncoated POPC/DOPE-MCC liposomes were mixed with $30 \%$ glycerol (v/v), frozen in liquid propane, and stored in liquid nitrogen until further use. Samples were fractured in a Balzers BAF400D freeze-etching apparatus (Balzers, Liechtenstein) under vacuum, with a pressure between $1.3 * 10^{-4}$ and $1.3 * 10^{-5} \mathrm{~Pa}$. Replicas were produced by vacuum deposition of the surface with platinum and carbon and controlled with a quartz crystal thin-film monitor. To clean the replicas, they were put into a sodium hypochlorite solution for about 3 hours and stored overnight in 50\% $\mathrm{NaOH}$. Before mounting them on an uncoated copper grid, replicas were washed with distilled water at least three times. Visualization of the grids was accomplished with the system already described, at an acceleration voltage of $50 \mathrm{kV}$.

\section{Release studies using ANTS/DPX}

Free ANTS/DPX was removed from ANTS/DPX-loaded liposomes by size exclusion chromatography using a Sephadex G75 column (Amersham Biosciences, Uppsala, Sweden). Subsequently, liposomes were coupled with chitosan-TGA at a 4:1 molar ratio (SH-groups:maleimide groups), or diluted with the same amount of deionized water (uncoupled liposomes). Release of ANTS/DPX from both liposomal suspensions was determined in simulated gastric fluid (SGF; $1 \mathrm{~L}$ contained $2 \mathrm{~g}$ of sodium chloride, $3.2 \mathrm{~g}$ of pepsin, and $7 \mathrm{~mL}$ of hydrochloric acid; $\mathrm{pH}$ 1.2) and simulated intestinal fluid (SIF; 1 L contained $6.8 \mathrm{~g}$ of monobasic potassium phosphate, $10 \mathrm{~g}$ of pancreatin, and $77 \mathrm{~mL}$ of $0.2 \mathrm{~N}$ sodium hydroxide; pH 6.8), which were prepared according to the US Pharmacopeial Convention. Samples were diluted 1:1 with one of these simulated body fluids and incubated for 24 hours. At fixed time points, $60 \mu \mathrm{L}$ of these mixtures was withdrawn, mixed with $2 \mathrm{~mL}$ of PBS, and measured fluorimetrically using a SPEX FLUOROMAX-3 fluorescence spectrometer (Jobin Yvon Horiba, Longjumeau Cedex, France) at an excitation wavelength of $360 \mathrm{~nm}$ and an emission wavelength of $530 \mathrm{~nm}$. To determine the fluorescence corresponding to a $100 \%$ release of ANTS/DPX, $10 \mu \mathrm{L}$ of $10 \%$ Triton $\mathrm{X}-100^{\mathrm{TM}}$ was added to the cuvette before measuring.

\section{Release studies using FITC-calcitonin}

Purification and coupling of chitosan-TGA to FITC-calcitoninloaded liposomes were performed in the same manner as for the ANTS/DPX-loaded liposomes. To determine the release of FITC-calcitonin from liposomes, a dialysis membrane was used to separate free from encapsulated peptide, as described previously by Saarinen-Savolainen et al. ${ }^{28}$ Briefly, $1 \mathrm{~mL}$ of freshly prepared drug-loaded liposomes, with or without chitosan-TGA coating, was mixed with $500 \mu \mathrm{L}$ of SGF or SIF, respectively. The mixture was transferred to a dialysis bag (molecular cut-off: $300 \mathrm{kDa}$ ), which was put into $15 \mathrm{~mL}$ of PBS solution. The solution outside the bag was stirred with a magnetic stirrer to ensure a continuous movement of the buffer solution and a homogenous distribution of released FITC-calcitonin. Samples of $800 \mu \mathrm{L}$ were withdrawn at fixed time intervals from the outer compartment and replaced immediately with equal volumes of PBS buffer solution. After 24 hours of testing, $15 \mu \mathrm{L}$ of $10 \%$ Triton $\mathrm{X}-100^{\mathrm{TM}}$ was added to the mixture inside the bag to disrupt the liposomes and release all of the entrapped peptide. This mixture was stirred for another 24 hours before determining the fluorescence of a $100 \%$ release. Samples were analyzed for FITC-calcitonin by measuring the fluorescence of $200 \mu \mathrm{L}$ of the withdrawn solution at an excitation wavelength of $490 \mathrm{~nm}$ and an emission wavelength of $525 \mathrm{~nm}$.

\section{In-vitro mucoadhesion studies}

For the mucoadhesion studies, we used a modified version of the falling liquid film technique previously described by Belgamwar et al. ${ }^{29} \mathrm{~A}$ freshly excised porcine small intestine was supplied by Karneta (Graz, Austria); $15 \mathrm{~cm}$ pieces were cut from the intestine and washed with physiological saline $\left(37^{\circ} \mathrm{C}\right)$. The intestinal tube was cut longitudinally, and a $5 \times 9 \mathrm{~cm}$ excised sheet was placed on a semicylindrical Plexiglas $^{\circledR}$ support with the mucosal side up (see Figure 2). The intestinal tissue was rinsed with $1 \mathrm{~mL}$ of rhodaminelabeled POPC or thiomer-coated liposomes (4:1 molar ratio of $\mathrm{SH}$-groups to maleimide groups) containing a total lipid amount of either 25 or $100 \mu \mathrm{g} / \mathrm{mL}$. The excess was collected in a petri dish and reapplied. This procedure was repeated ten times and the fluorescence intensity of the final residual solution $\left(I_{t}\right)$ was measured with a fluorimeter (FluoStar Galaxy; LABTECH, Offenburg, Germany) 

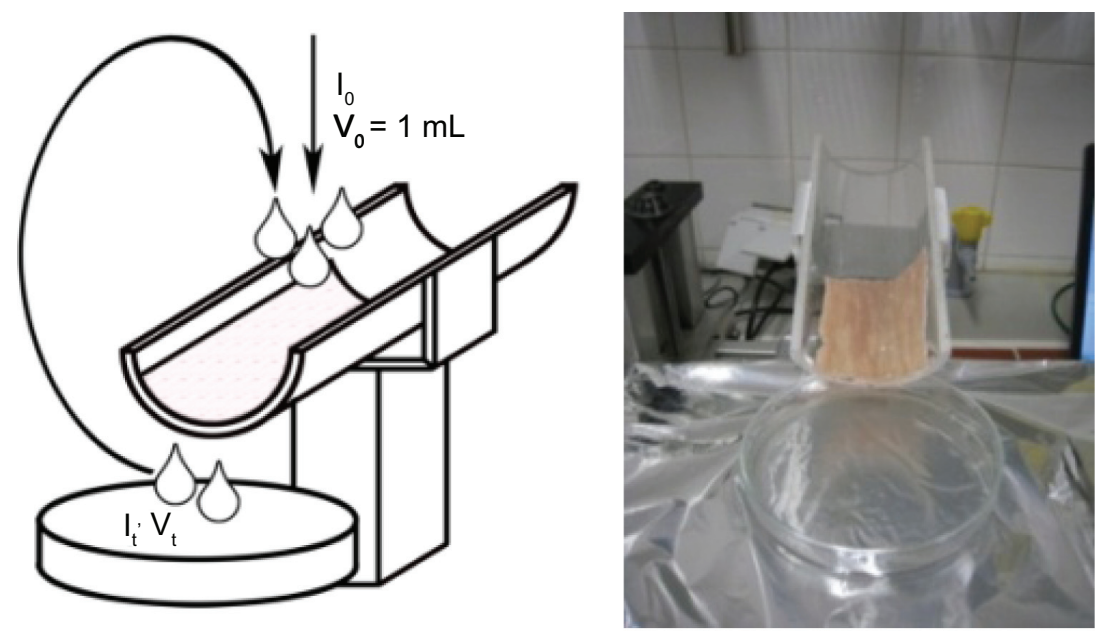

Figure 2 Falling Liquid Film technique to measure the mucoadhesion of coated and uncoated liposomes.

at an excitation wavelength of $544 \mathrm{~nm}$ and an emission wavelength of $590 \mathrm{~nm}$. A 96-well plate $\left(\right.$ Cellstar $^{\circledR}$, Greiner Bio-One GmbH, Friedrichshafen, Germany) was used throughout the study. The mean value of at least three measurements was determined to calculate the amount of bound liposomes according to the following equation:

$$
\text { Adhesive } \%=\frac{\left(I_{0} * V_{0}\right)-\left(I_{t} * V_{t}\right)}{\left(I_{0} * V_{0}\right)} * 100
$$

where $I_{0}$ is the fluorescence of the initial solution, $V_{0}$ is the volume that is applied to the tissue; $V_{t}$ is the final volume of the unbound solution; and Adhesive \% corresponds to the adhesive fraction of the applied liposomal suspension.

\section{Cell viability}

To make sure that the intestinal tissues used for these experiments were still viable, cell vitality assays were carried out using 3-(4,5-dimethylthiazol-2-yl)-2,5-diphenyltetrazolinum bromide (MTT). ${ }^{30}$ Consequently, tissues were sliced into $5 \mathrm{~mm}$ samples after extensively washing them $(n=12)$. Half of the samples were boiled in water for 1 hour to inactivate the enzymes (zero value). All excidates were transferred to a 12 -well plate containing $2 \mathrm{~mL}$ of MTT solution $(2 \mathrm{mg} / \mathrm{mL})$, and were incubated for 2 hours at $37^{\circ} \mathrm{C}, 95 \%$ air $/ 5 \% \mathrm{CO}_{2}$ in the incubator. Subsequently, the MTT was removed and the samples were washed twice with $1 \mathrm{~mL}$ of PBS. The samples were then minced with scissors and the formazan precipitate was extracted in $4 \mathrm{~mL}$ of dimethyl sulfoxide for 80 minutes on a rotating platform (80 rpm; Orbital Schuttler OD 10C; Al-Labortechnik, Amstetten, Austria). The formazan absorbance was recorded at $544 \mathrm{~nm}$, with dimethyl sulfoxide as blank, on a plate reader.

\section{Fluorescence microscopy}

After the application of liposomes, the intestinal tissue was incubated for 30 minutes at $37^{\circ} \mathrm{C} / 95 \%$ air $/ 5 \% \mathrm{CO}_{2}$ and fixed with 4\% formalin for 3-4 hours at room temperature. Tissues were embedded in Tissue Tec OCT (Sanova, Vienna, Austria) and $10 \mu \mathrm{m}$ sections were cut using a HM560 Cryostat (Thermo Scientific, Walldorf, Germany). To assess tissue penetration, bright field images and fluorescent images - with excitation BP 520-550 $\mathrm{nm}$ and emission LP $580 \mathrm{~nm}$ for red fluorescence - were acquired with an Olympus BX-51 microscope (Olympus, Vienna, Austria). Sections with tissue not exposed to liposomes (negative controls) were used to evaluate autofluorescence.

\section{Cytotoxicity screening}

Human colorectal adenocarcinoma cells were used for cytotoxicity testing; $2 \times 10^{4}$ cells/well were seeded 24 hours before treatment in 96-well plates, exposed to thiomer-coated liposomes (4:1 molar ratio of SH-groups to maleimide groups), and suspended in Dulbecco's modified Eagle medium in concentrations of $0-1000 \mu \mathrm{g} / \mathrm{mL}$. Exposures were performed at $37^{\circ} \mathrm{C}$ in a $95 \%$ air $/ 5 \% \mathrm{CO}_{2}$ atmosphere, and two time points (4 and 24 hours) were evaluated. Contamination with endotoxin was assessed via a PYROGENT ${ }^{\circledR}$ Ultra Gel Clot Limulus Amoebocyte Lysate Assay (LONZA, Vienna, Austria). Only low concentrations of endotoxin were detected at the highest sample concentration. Cytotoxicity was studied by formazan bioreduction (CellTiter 96 ${ }^{\circledR}$ AQueous Non-Radioactive Cell Proliferation Assay; Promega, Vienna, Austria); ATP content (CellTiter-Glo Luminescent Cell Viability Assay; Promega); and membrane integrity (CytoTox-ONE ${ }^{\mathrm{TM}}$ 
Homogeneous Membrane Integrity Assay; Promega), as previously described. ${ }^{31}$

\section{Statistical analysis}

Data are presented as mean \pm standard deviation. Data sets were compared using Student's $t$-test and differences were considered as significant at $P<0.05$.

\section{Results and discussion Preparation and characterization of thiomer-coated liposomes}

To develop lipid nanoparticles with improved mucoadhesive properties, we explored the potential of thiolated polymers to coat the surface of preformed liposomes. For this purpose, we used low-molecular-weight chitosan, which had previously been modified by thioglycolic acid, as described by Kast et al. ${ }^{17}$ Chitosan-TGA is characterized by $539 \pm 57 \mu \mathrm{mol}$ free-SH groups/g polymer and $72 \pm 38 \mu \mathrm{mol}$ disulfide bridges/g polymer. An earlier study showed that nanoparticles formulated by such thiolated chitosans revealed significantly higher mucoadhesive properties than unmodified chitosan nanoparticles. ${ }^{19}$ In the present study, we covalently linked some of the free SH-groups of chitosan-TGA to maleimidegroups - provided by functionalized phospholipid molecules (DOPE-MCC) - which are part of the liposome bilayer and make approximately $10 \mathrm{~mol} \%$ of total lipids (Figure 1). As controls, liposomes without functionalized phospholipids were incubated with the same amount of polymer.

\section{Size distribution}

The size of the unmodified particles was determined to be $167 \mathrm{~nm}(\mathrm{n}=3)$ for POPC/DOPE-MCC liposomes and $195 \mathrm{~nm}$ $(n=3)$ for liposomes containing only POPC. The polydispersity index values were in the range of $0.1-0.2$, displaying one homogeneous population of liposomes with a narrow size distribution. The difference in the size of POPC liposomes and POPC/DOPE-MCC liposomes is likely to be caused by the presence of the maleimide-group on the head region of the DOPE lipid. With an increasing amount of polymer, given by the molar ratio of $\mathrm{SH}$-groups to maleimide-groups, the size of the POPC/DOPE-MCC liposomes gradually increased. Above a ratio of $2: 1$, the curve levels off and only small increases are detectable as more polymer is added (Figure 3). No significant change in size was detectable for control liposomes. The polydispersity of POPC/DOPEMCC liposomes increased after coupling to a value of $\sim 0.5$ compared with $\sim 0.2$ for control liposomes. This indicates a

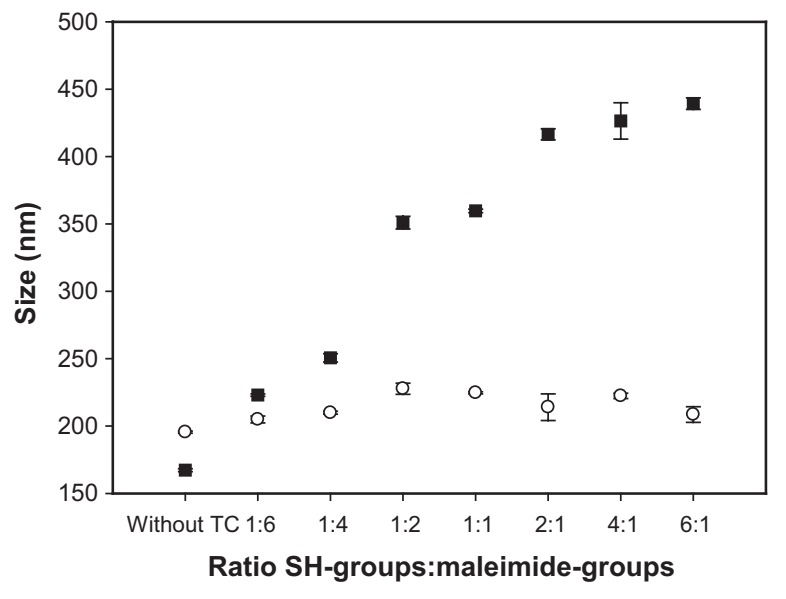

Figure 3 Particle size after the addition of different amounts of polymers (given by the molar ratio of $\mathrm{SH}$-groups of the polymer to maleimide groups of the liposome) to POPC liposomes (O) and POPC/DOPE-MCC liposomes ( $\square$ ).

Note: Indicated values are means \pm standard deviation of at least three measurements. Abbreviations: DOPE-MCC, I,2-dioleoyl-sn-glycero-3-phosphoethanolamine-N[4-(p-maleimidomethyl)cyclohexane-carboxamide]; POPC, Palmitoyl-oleoylphosphatidylcholine; TC, chitosan-thioglycolic acid.

more heterogeneous size distribution of coupled relative to uncoupled liposomes.

To test whether the increase in particle size was caused by one polymer chain binding to more than one liposome, or by intermolecular disulfide bridges between free SH-groups of the chitosan-TGA polymer coat, we added a reducing agent before measuring particle size. As we found no differences in the size values before and after reduction, size increase caused by disulfide formation is highly unlikely.

\section{Zeta potential}

To investigate the influence of the polymer coating on the surface charge of liposomes, the zeta potential was measured. The negatively charged phosphate group of the functionalized lipid is responsible for the highly negative zeta potential of uncoated POPC/DOPE-MCC liposomes, whereas control liposomes consisting only of POPC are roughly neutrally charged (Figure 4 ). The zeta potential of maleimide-functionalized liposomes increased from $-38 \mathrm{mV}$ to $+20 \mathrm{mV}$, with increasing polymer concentration. The polymer itself is positively charged, and coupling of the polymer to the liposome increased the potential of the resulting particle. After the addition of the smallest amount of polymer used in this study, a rapid increase in the zeta potential was observed. Then, the zeta potential slightly increased - coinciding with the increasing amount of polymer - and became positive, reaching a plateau at a $4: 1$ ratio of $\mathrm{SH}$-groups to maleimide-groups. This observation confirms the results obtained from the size measurements, 


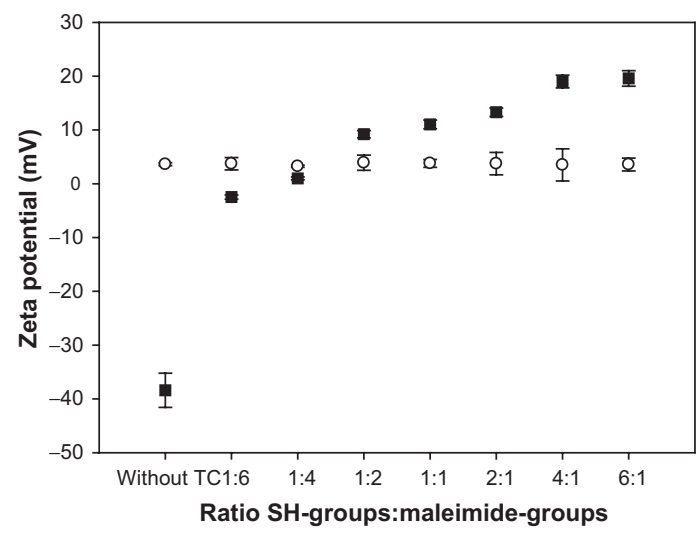

Figure 4 Zeta potential after the addition of different amounts of polymers (given by the molar ratio of $\mathrm{SH}$-groups of the polymer to maleimide groups of the liposome)

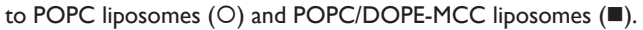

Note: Indicated values are means \pm standard deviation of at least three measurements. Abbreviations: DOPE-MCC, I,2-dioleoyl-sn-glycero-3-phosphoethanolamine$\mathrm{N}$-[4-(p-maleimidomethyl)cyclohexane-carboxamide]; POPC, Palmitoyl-oleoylphosphatidylcholine; TC, chitosan-thioglycolic acid.

indicating a polymer saturation concentration. Therefore, a ratio of 4:1 was chosen for mucoadhesion and drug release studies. In contrast to POPC/DOPE-MCC liposomes, no change was measurable after adding different amounts of polymer to pure POPC liposomes.

To summarize, the change in the zeta potential from highly negative to positive values and the increase in the size of the liposomes by about $270 \mathrm{~nm}$, led us to conclude that the polymer is successfully linked to the liposomal surface. This increase after coupling correlates well with the observations of other groups. ${ }^{32-35}$ The polymer is most likely bound to the liposomal surface by no other force than the covalent bond, since no effect of the polymer on the size or zeta potential of control liposomes could be detected.

\section{Determination of free $\mathrm{SH}$-groups}

The amount of free $\mathrm{SH}$-groups was measured after previous incubation of the samples with $\mathrm{NaBH}_{4}$ to reduce disulfide bridges, which may be formed between thiol groups. After coupling, we found $283 \mathrm{nmol}$ free SH-groups/mg lipid, for a coupling ratio of $4: 1$ and about $494 \mathrm{nmol} \mathrm{SH-groups} / \mathrm{mg}$ lipid, for a coupling ratio of 6:1 (SH-groups to maleimide groups) (see Table 1). When applied to the small intestine, the polymer, which is bound to the surface of liposomes after coupling, is able to interact with cysteine residues of mucus glycoproteins via freely available SH-groups. Consequently, thiomer-coated liposomes become covalently attached to the intestinal mucus by disulfide bridges. This should lead to an improved mucoadhesion of the particles. ${ }^{16}$

For maleimide-functionalized liposomes, we found $\sim 100 \mathrm{nmol} \mathrm{SH}$-groups $/ \mathrm{mg}$ lipid less than for control liposomes.
Table I Amount of SH-groups after coupling

\begin{tabular}{lllll}
\hline & $\begin{array}{l}\text { Coupled } \\
\text { liposomes } \\
4: 1\end{array}$ & $\begin{array}{l}\text { Control } \\
\text { liposomes } \\
4: 1\end{array}$ & $\begin{array}{l}\text { Coupled } \\
\text { liposomes } \\
6: 1\end{array}$ & $\begin{array}{l}\text { Control } \\
\text { liposomes } \\
\text { 6:1 }\end{array}$ \\
\hline $\begin{array}{l}\text { Amount of } \\
\text { SH-groups }\end{array}$ & $283 \pm 40$ & $382 \pm 19$ & $494 \pm 29$ & $594 \pm 12$ \\
(nmol/mg & & & & \\
total lipid) & & & & \\
\hline
\end{tabular}

Notes: Coupled liposomes (POPC/DOPE-MCC) with a coupling ratio (SH-groups to maleimide groups) of 4:I and 6:I, and control liposomes (POPC) with the same amount of polymer solution added. All values are means \pm standard deviation of five experiments.

Abbreviations: DOPE-MCC, 1,2-dioleoyl-sn-glycero-3-phosphoethanolamine$\mathrm{N}$-[4-(p-maleimidomethyl)cyclohexane-carboxamide]; POPC, Palmitoyl-oleoylphosphatidylcholine.

This reduction in the amount of free SH-groups after coupling is a strong indication for a successful binding of chitosanTGA to liposomes via maleimide groups. This difference of $100 \mathrm{nmol} \mathrm{SH-groups/mg} \mathrm{lipid} \mathrm{did} \mathrm{not} \mathrm{change} \mathrm{by} \mathrm{increasing}$ the coupling ratio, as this difference represents the amount of maleimide groups in the sample, to which free SH-groups were coupled.

\section{Liposome morphology}

To study the morphology of coated and uncoated liposomes, negative staining transmission electron microscopy was chosen. Ammonium molybdate (5\%), phosphotungstic acid $(1 \%)$, and uranyl acetate $(2 \%)$ were tried as staining agents. Uranyl acetate was found to give the best contrast and the most homogeneous distribution of the dye and was used for all further preparations. Pictures of uncoated liposomes showed mostly spherical liposomes (170-200 nm mean size). After adding the polymer, the liposomes were apparently linked with each other (Figure 5). With this technique, we were able to rule out the possibility that the increase in size of the liposomes, after adding the polymer, was caused by fusion. Nevertheless, we were not able to detect the polymer coat itself in negative-contrasted samples. Therefore, freeze fracturing was used to reveal the polymer coat (Figure 6). Drying and staining steps, during which the liposomes may suffer from different $\mathrm{pH}$ or salt conditions, are not necessary; however, images prepared by freeze fracturing do not reflect the real size of the liposomes, since fracturing does not have to occur through the center of the particles. ${ }^{36}$ The freeze fractures of the coated samples clearly revealed the presence of the polymer at the periphery of the liposomes, which is marked by arrows in Figure 6B. Although no association of liposomes was found in uncoated samples (Figure 6A), single liposomes - as well as aggregates, which involve about three to ten liposomes - were visible 

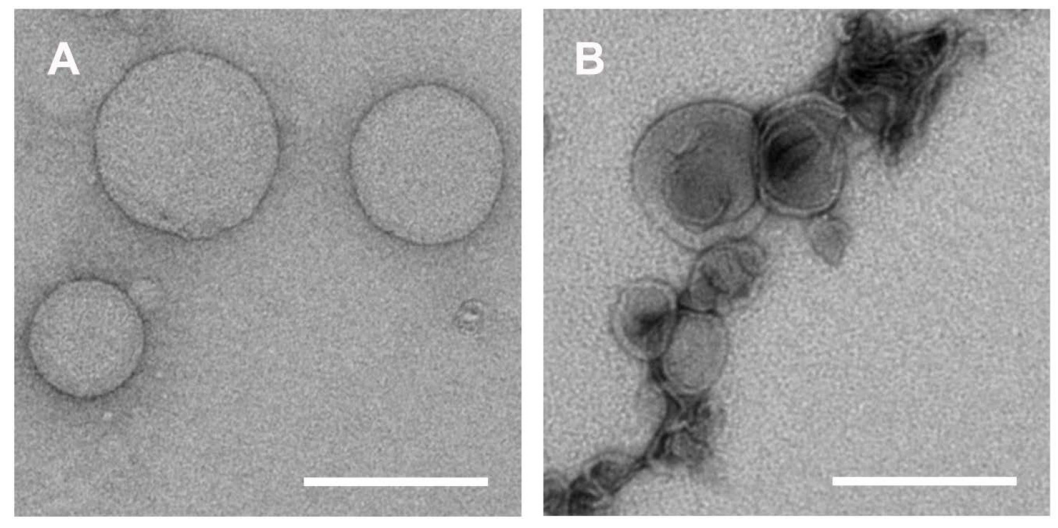

Figure 5 Transmission electron micrographs with negative staining technique of (A) uncoated POPC/DOPE-MCC liposomes and (B) POPC/DOPE-MCC liposomes coated with chitosan-TGA (4:I molar ratio of $\mathrm{SH}$-groups to maleimide groups).

Notes: Magnification: 30,000×. Scale bar indicates $200 \mathrm{~nm}$.

Abbreviations: DOPE-MCC, I,2-dioleoyl-sn-glycero-3-phosphoethanolamine-N-[4-(p-maleimidomethyl)cyclohexane-carboxamide]; POPC, Palmitoyl-oleoyl-phosphatidylcholine; TGA, thioglycolic acid.

in the coated sample (Figure 6B). Henriksen et al observed equally heterogeneous aggregates after adding negatively charged liposomes to a chitosan solution using cryo-electron microscopy. ${ }^{35}$ This heterogeneity is also reflected in the polydispersity index of the size measurements, which increased with increasing the amount of polymer.

Aggregates between liposomes and the polymer can be formed by different mechanisms. One possibility is that one polymer chain binds to different liposomes, thereby crosslinking single particles. This mechanism was also proposed by Mertins et al ${ }^{37}$ where they bound chitosan to preformed liposomes by ionic interactions. Another possibility would be an entanglement of polymer chains that were previously bound to different liposomes. Disulfide formation has already been ruled out, as previously stated. Neither negative staining nor freeze-fracture electron microscopy allowed us to differentiate between these scenarios. However, since aggregates are still present at very high dilutions, the first mechanism is more likely.

\section{Drug encapsulation and release}

To study drug loading and release profiles of thiomer-coated liposomes, we tested two different model drug systems. First, the fluorophore/quencher couple ANTS/DPX was used. Second, the fluorescent-labeled peptide calcitonin (FITCcalcitonin) was encapsulated with an entrapment efficiency of $21.5 \%$, which gave a final peptide concentration of $1.4 \mu \mathrm{g} /$ mg lipid.

The ANTS/DPX assay is usually performed to measure the influence of different agents on membrane stability. ${ }^{38}$
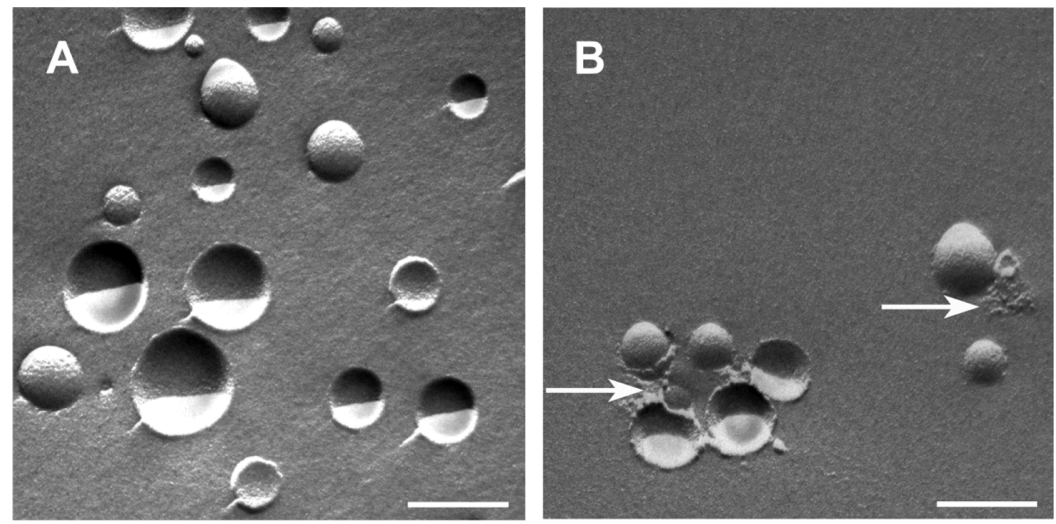

Figure 6 Transmission electron micrographs using freeze fracturing of (A) uncoated POPC/DOPE-MCC liposomes and (B) POPC/DOPE-MCC liposomes coated with chitosan-TGA (4:I molar ratio of $\mathrm{SH}$-groups to maleimide groups).

Notes: Arrows indicate the polymer coat. Magnification: 30,000x. Scale bar indicates $200 \mathrm{~nm}$.

Abbreviations: DOPE-MCC, I,2-dioleoyl-sn-glycero-3-phosphoethanolamine-N-[4-(p-maleimidomethyl)cyclohexane-carboxamide]; POPC, Palmitoyl-oleoyl-phosphatidylcholine; TGA, thioglycolic acid. 
Hence, this system seems very suitable to quantify drug release. ${ }^{39}$ In principle, both the fluorophore ANTS and the quencher molecule DPX are encapsulated in liposomes during the hydration step. The molecules are in close proximity to each other and the short distance between the quencher and fluorophore leads to a quenching of the latter; consequently, the monitored fluorescence intensity is low. Upon leakage and release of ANTS/DPX, the fluorescence intensity increases in a concentration-dependent manner. With this technique, we could monitor the release profile from coupled and uncoupled liposomes at different environmental conditions. In particular, we investigated the release properties in simulated gastric and intestinal fluids. Released ANTS/DPX can be measured at fixed time points without separating the released fluorophore. In the second model drug system, we separated released from encapsulated FITC-calcitonin by dialysis before measuring the fluorescence.

The release of ANTS/DPX (Figure 7) corresponded well with the release of FITC-calcitonin (Figure 8); albeit, the release of FITC-calcitonin in SGF was slightly higher than the release of ANTS/DPX in the same medium. Comparing the release within the first 2 hours, it was for all tested liposomes and model drugs in both SGF and SIF about $25 \%$ $30 \%$. Even though there was no significant difference in the release profiles of model drugs within the first few hours, we observed a slightly higher release from thiomer-coated liposomes after 24 hours (Figures 7 and 8). To further investigate this effect, coupled and uncoupled liposomes containing either ANTS/DPX or FITC-calcitonin were kept in PBS and measured after 24 hours. For ANTS/DPX, the released fraction was $38 \%$ for coated liposomes compared with $10 \%$ for uncoated liposomes, which were just mixed with water. The release behavior of FITC-calcitonin was similar, showing $39 \%$ and $13 \%$ for coated and uncoated liposomes, respectively. In both cases, a higher release of encapsulated compounds was found for thiomer-coated liposomes in the buffer. Polymer linkage apparently induced this increased release, suggesting the occurrence of some defects in the bilayer, which could be explained by force being exerted on phospholipid head-groups by intermolecular crosslinking. These results suggest that coated liposomes are, by themselves, more leaky, due to the polymer coat. Nonetheless, they are not less stable in simulated body fluid than uncoated ones. Nevertheless, neither coated nor uncoated liposomes released the encapsulated compounds abruptly, nor were they immediately disrupted by the enzymes - a fact that is crucial for a sustained release system.

\section{In vitro mucoadhesion}

To mimic physiological conditions for all experiments, the viability of the membrane, which represents a key parameter for reproducible data, was evaluated. The results revealed that the viability decreased as time increased, indicating that the mucosa has to be used immediately. The mucoadhesiveness of surface-modified liposomes was investigated using the falling liquid film technique (see Figure 2). By measuring the fluorescence of the particle solution before and after applying it to the mucus of the porcine small intestine, we could determine differences in the mucoadhesion of chitosan-TGA-coated and uncoated liposomes.
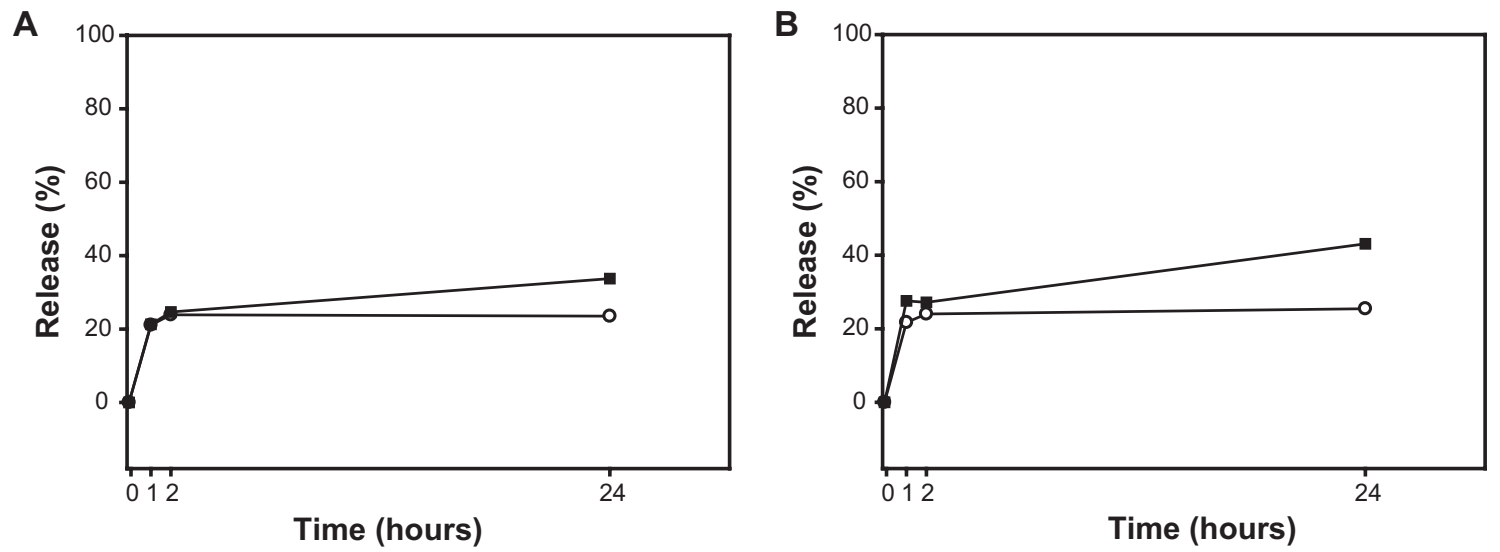

Figure 7 Release of ANTS/DPX at predetermined time points from uncoated POPC/DOPE-MCC liposomes (O) and coated POPC/DOPE-MCC liposomes (4:I molar ratio of $\mathrm{SH}$-groups to maleimide groups) ( $\mathbf{\square})$ in simulated gastric fluid $(\mathbf{A})$ and simulated intestinal fluid (B).

Note: Each point represents the mean value of two different determinations.

Abbreviations: ANTS, anionic fluorophore 8-aminonaphthalene-I,3,6-trisulfonic acid; DOPE-MCC, I,2-dioleoyl-sn-glycero-3-phosphoethanolamine-N-[4-(p-maleimidomethyl) cyclohexane-carboxamide]; DPX, p-xylene-bis-pyridinium bromide; POPC, Palmitoyl-oleoyl-phosphatidylcholine. 

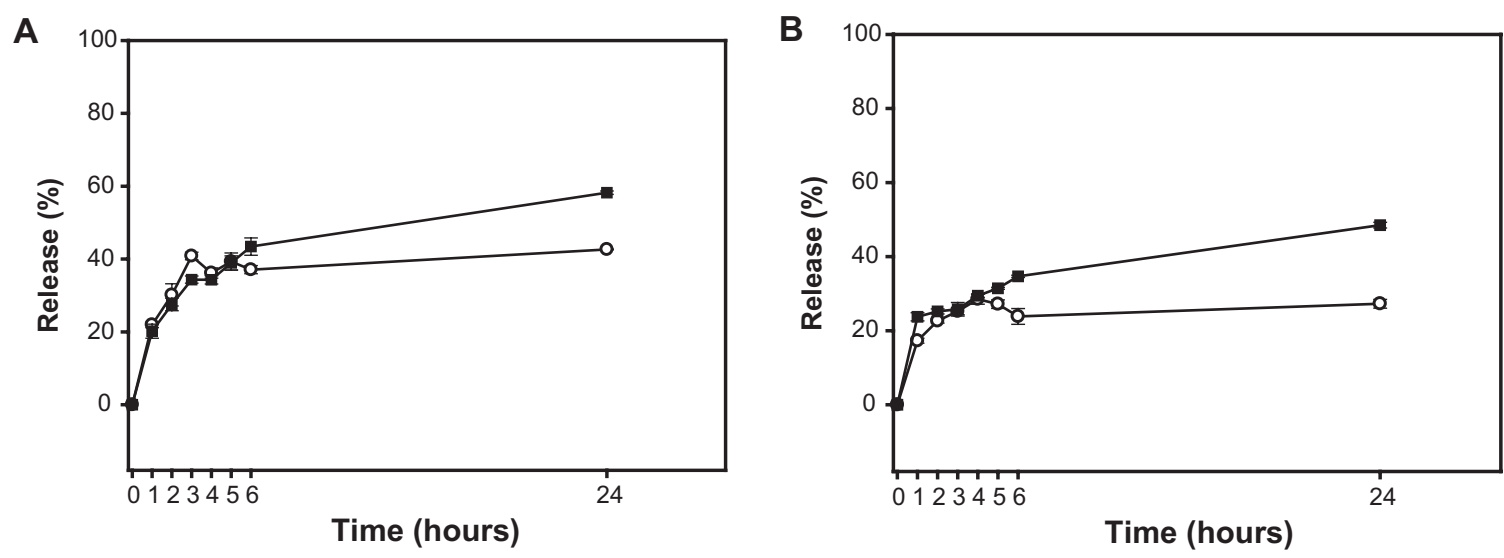

Figure 8 Release of FITC-calcitonin at predetermined time points from uncoated POPC/DOPE-MCC liposomes (O) and coated POPC/DOPE-MCC liposomes (4:I molar ratio of $\mathrm{SH}$-groups to maleimide groups) $(\mathbf{\square})$ in simulated gastric fluid $(\mathbf{A})$ and simulated intestinal fluid (B).

Note: Results are means \pm standard deviation $(n=3)$.

Abbreviations: DOPE-MCC, I,2-dioleoyl-sn-glycero-3-phosphoethanolamine-N-[4-(p-maleimidomethyl)cyclohexane-carboxamide]; POPC, Palmitoyl-oleoyl-phosphatidylcholine.

Thiomer-coated liposomes showed a $\sim 1.8$-fold higher mucoadhesive effect relative to the uncoated lipsomes. The improvement ratio of coated to uncoated liposomes was not significantly different, whether 25 or $100 \mu \mathrm{g}$ of liposomes was added to the tissue (Table $2 \mathrm{~A}$ and $\mathrm{B}$ ).

To visualize the adhered liposomes, the treated tissues were viewed by fluorescence microscopy (Figure 9). The results demonstrate that tissues incubated with coated liposomes clearly showed a higher concentration of rhodamine-labeled particles than tissues exposed to uncoated liposomes. Additionally, we observed a tight adherence of the coated liposomes to the surface of the intestinal mucus. The fluorescence microscopic images illustrate that the intestinal tissue seems to be affected by the procedure; via formalin fixation and paraffin embedding, we could show that the integrity of the epithelial layer is preserved (data not shown).

Table 2 Percentage of uncoated/coated liposomes bound to the tissue after using the falling liquid film technique

\begin{tabular}{lll}
\hline $\begin{array}{l}\text { Mucoadhesion } \\
\text { of coated }\end{array}$ & $\begin{array}{l}\text { Mucoadhesion } \\
\text { of uncoated } \\
\text { liposomes (\%) }\end{array}$ & $\begin{array}{l}\text { Improvement } \\
\text { ratio }\end{array}$ \\
\hline
\end{tabular}

\begin{tabular}{lll}
\hline (A) $25 \mu \mathrm{g} / \mathrm{mL}$ & & \\
70.4 & 33.5 & 2.1 \\
68.4 & 46.1 & 1.5 \\
61.3 & 41.7 & 1.5 \\
(B) $100 \mu \mathrm{g} / \mathrm{mL}$ & & \\
56.6 & 31.4 & 1.8 \\
60.3 & 45.0 & 1.3 \\
60.0 & 26.0 & 2.3 \\
\hline
\end{tabular}

Notes: Comparison of three independent tests; $\mathrm{I} \mathrm{mL}$ of coated or uncoated liposomes with a concentration of (A) $25 \mu \mathrm{g} / \mathrm{mL}$ or (B) $100 \mu \mathrm{g} / \mathrm{mL}$ was applied to the tissue and the amount of bound particles was calculated as a percentage (\%) of the total.
Although various groups could show a strong electrostatic interaction between chitosan and liposomes, a covalent bond is generally considered more stable than electrostatic surface interactions. In terms of mucoadhesion, this might be advantageous, since the whole liposome-polymer complex is bound to the mucosal membrane. The observed positive zeta potential of thiomer-coated liposomes could also contribute to an improved mucoadhesion due to ionic interactions between the positively charged polymer and the negatively charged constituents of the mucus layer; that is, sulfonic and sialic acid residues. ${ }^{40,41}$ Moreover, thiolated polymers, such as the chitosan-TGA used for our experiments, form disulfide bridges with cysteine-rich subdomains of mucus glycoproteins. ${ }^{5}$ Thus, thiomer-coated liposomes can be attached covalently to the intestinal mucus, where the encapsulated compound is sustainedly released, as was shown by our release studies. Even though electrostatically chitosan-coated liposomes show a high mucoadhesion ${ }^{42,43}$ it is hard to judge whether the polymer stays attached to the liposomal surface throughout the whole gastrointestinal transit.

\section{Cytotoxicity screening}

For the assessment of cytotoxicity, the metabolic functions, according to formazan bioreduction and cellular ATP content, were determined. In addition, cell membrane integrity was assessed by the release of lactate dehydrogenase. In all three assays (up to $1 \mathrm{mg} / \mathrm{mL}$ of thiomer-coated liposomes), no indication of cellular damage was seen after incubation for 4 and 24 hours. Consistent with these results, cell membrane integrity, which was verified by the absence of lactate 

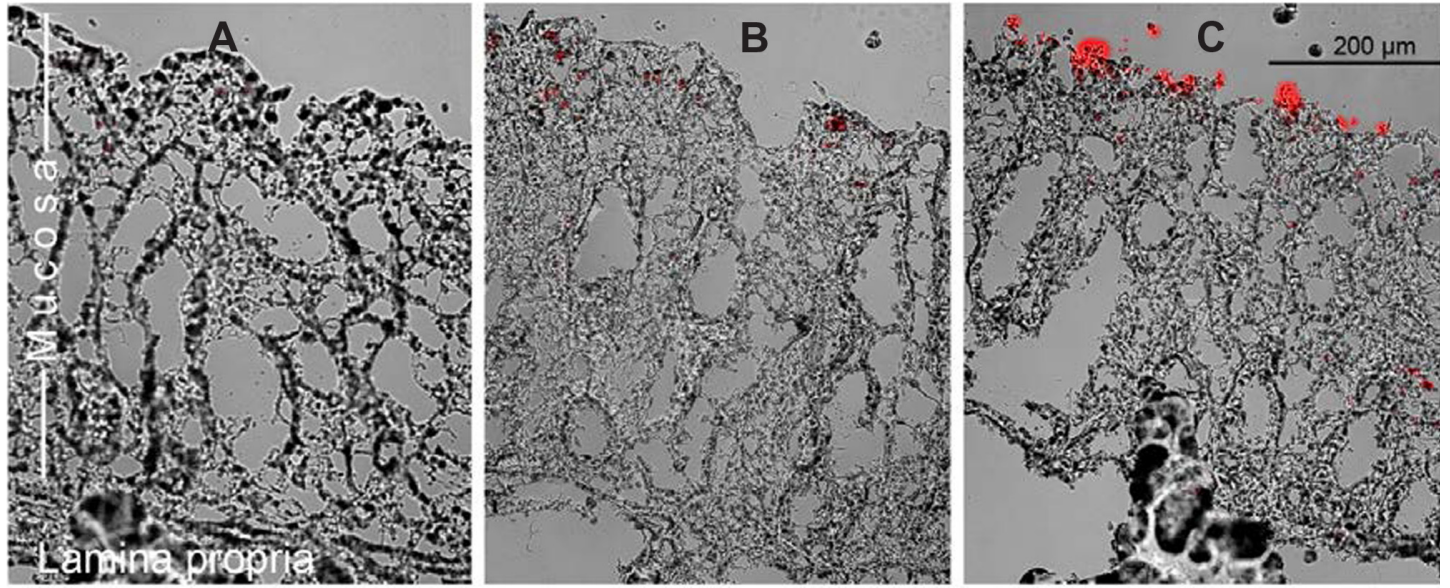

Figure 9 Histological sections of porcine small intestine. (A) untreated intestinal tissue (negative control), (B) tissue treated with POPC/DOPE-Liss Rhod liposomes and (C) with POPC/DOPE-Liss Rhod/DOPE-MCC liposomes coated with chitosan-TGA (molar ratio of SH-groups to maleimide-groups: 4:I).

Abbreviations: DOPE-Liss Rhod, I,2-dioleoyl-sn-glycero-3-phosphoethanolamine-N-(lissamine rhodamine B sulfonyl); DOPE-MCC, I,2-dioleoyl-sn-glycero-3phosphoethanolamine-N-[4-(p-maleimidomethyl)cyclohexane-carboxamide]; POPC, Palmitoyl-oleoyl-phosphatidylcholine; TGA, thioglycolic acid.

dehydrogenate release, was maintained. Taken together, these results demonstrate the absence of cytotoxic effects in the concentration range tested.

\section{Conclusion}

A novel delivery system, based on the coating of liposomes with thiolated chitosan, has been successfully synthesized and characterized. In contrast to the common coating procedure, in which polymers are attached to liposomes by ionic interactions, we have established a covalent thioether bond between chitosan-TGA and the liposome. This covalent coupling was confirmed by size and zeta potential measurements, as well as negative-staining transmission electron microscopy images. In vitro mucoadhesion studies of thiomer-coated and uncoated liposomes showed that the residence time on porcine small intestine could be almost doubled by the addition of the polymeric layer around the liposome. Based on these investigations, we propose that coating liposomes with chitosan-TGA represents a promising strategy to create a mucoadhesive oral delivery system for sustained drug release.

\section{Acknowledgments/Disclosure}

This work was supported by the Austrian Nano-Initiative, which cofinanced this work as part of the Nano-Health Project (no 819721), financed by the Austrian Research Promotion Agency (FFG). For technical assistance with electron microscopy, we would like to thank Elisabeth Bock and Gertrud Havlicek. For assistance with mucoadhesion studies, we would like to thank Christina Judmaier and Dagmar Stratil. The authors report no conflicts of interest in this work.

\section{References}

1. Lasic DD, Papahadjopoulos D. Medical Applications of Liposomes. Amsterdam: Elsevier Science; 1998.

2. Allen TM, Cullis PR. Drug delivery systems: entering the mainstream. Science. 2004;303(5665):1818-1822.

3. Torchilin VP. Recent advances with liposomes as pharmaceutical carriers. Nat Rev Drug Discov. 2005;4(2):145-160.

4. Patel HM, Stevenson RW, Parsons JA, Ryman BE. Use of liposomes to aid intestinal absorption of entrapped insulin in normal and diabetic dogs. Biochim Biophys Acta. 1982;716(2):188-193.

5. Chiang CM, Weiner N. Gastrointestinal uptake of liposomes. II. In vivo studies. Int J Pharm. 1987;40(1-2):143-150.

6. Parmentier J, Hartmann FJ, Fricker G. In vitro evaluation of liposomes containing bio-enhancers for the oral delivery of macromolecules. Eur J Pharm Biopharm. 2010;76(3):394-403.

7. Takeuchi H, Matsui Y, Sugihara H, Yamamoto H, Kawashima Y Effectiveness of submicron-sized, chitosan-coated liposomes in oral administration of peptide drugs. Int J Pharm. 2005;303(1-2):160-170.

8. Takeuchi H, Matsui Y, Yamamoto H, Kawashima Y. Mucoadhesive properties of carbopol or chitosan-coated liposomes and their effectiveness in the oral administration of calcitonin to rats. $J$ Control Release. 2003;86(2-3):235-242.

9. Karn PR, Vanic Z, Pepic I, Skalko-Basnet N. Mucoadhesive liposomal delivery systems: the choice of coating material. Drug Dev Ind Pharm. 2011;37(4):482-488.

10. Dwivedi N, Arunagirinathan MA, Sharma S, Bellare J. Silica-coated liposomes for insulin delivery. $J$ Nanomater. 2010;2010:Article ID 652048.

11. Werle M, Takeuchi H. Chitosan-aprotinin coated liposomes for oral peptide delivery: Development, characterisation and in vivo evaluation. Int J Pharm. 2009;370(1-2):26-32.

12. Longer MA, Ch'ng HS, Robinson JR. Bioadhesive polymers as platforms for oral controlled drug delivery III: oral delivery of chlorothiazide using a bioadhesive polymer. J Pharm Sci. 1985;74(4): 406-411.

13. Zaru M, Manca ML, Fadda AM, Antimisiaris SG. Chitosan-coated liposomes for delivery to lungs by nebulisation. Colloids Surf B Biointerfaces. 2009;71(1):88-95.

14. Li N, Zhuang C, Wang M, Sun X, Nie S, Pan W. Liposome coated with low molecular weight chitosan and its potential use in ocular drug delivery. Int J Pharm. 2009;379(1):131-138. 
15. Amin M, Jaafari MR, Tafaghodi M. Impact of chitosan coating of anionic liposomes on clearance rate, mucosal and systemic immune responses following nasal administration in rabbits. Colloids Surf B Biointerfaces. 2009;74(1):225-229.

16. Bernkop-Schnurch A, Steininger S. Synthesis and characterisation of mucoadhesive thiolated polymers. Int J Pharm. 2000;194(2): 239-247.

17. Kast CE, Bernkop-Schnurch A. Thiolated polymers - thiomers: development and in vitro evaluation of chitosan-thioglycolic acid conjugates. Biomaterials. 2001;22(17):2345-2352.

18. Millotti G, Samberger C, Frohlich E, Bernkop-Schnurch A. Chitosangraft-6-mercaptonicotinic acid: synthesis, characterization, and biocompatibility. Biomacromolecules. 2009;10(11):3023-3027.

19. Bernkop-Schnurch A, Weithaler A, Albrecht K, Greimel A. Thiomers: preparation and in vitro evaluation of a mucoadhesive nanoparticulate drug delivery system. Int J Pharm. 2006;317(1):76-81.

20. Bernkop-Schnurch A, Scholler S, Biebel RG. Development of controlled drug release systems based on thiolated polymers. $J$ Control Release. 2000;66(1):39-48.

21. Barthelmes J, Dunnhaupt S, Hombach J, Bernkop-Schnurch A. Thiomer nanoparticles: stabilization via covalent cross-linking. Drug Deliv. 2011;18(8):613-619.

22. Bernkop-Schnurch A, Kast CE, Guggi D. Permeation enhancing polymers in oral delivery of hydrophilic macromolecules: thiomer/GSH systems. J Control Release. 2003;93(2):95-103.

23. Illum L. Chitosan and its use as a pharmaceutical excipient. Pharm Res. 1998;15(9):1326-1331.

24. Takeuchi H, Thongborisute J, Matsui Y, Sugihara H, Yamamoto H, Kawashima Y. Novel mucoadhesion tests for polymers and polymercoated particles to design optimal mucoadhesive drug delivery systems. Adv Drug Deliv Rev. 2005;57(11):1583-1594.

25. Laye C, McClements DJ, Weiss J. Formation of biopolymer-coated liposomes by electrostatic deposition of chitosan. J Food Sci. 2008;73(5): N7-N15.

26. Mady MM, Darwish MM, Khalil S, Khalil WM. Biophysical studies on chitosan-coated liposomes. Eur Biophys J. 2009;38(8):1127-1133.

27. Bravo-Osuna I, Schmitz T, Bernkop-Schnurch A, Vauthier C, Ponchel G. Elaboration and characterization of thiolated chitosancoated acrylic nanoparticles. Int J Pharm. 2006;316(1-2): $170-175$.

28. Saarinen-Savolainen P, Jarvinen T, Taipale H, Urtti A. Method for evaluating drug release from liposomes in sink conditions. Int J Pharm. 1997;159(1):27-33.

29. Belgamwar V, Shah V, Surana SJ. Formulation and evaluation of oral mucoadhesive multiparticulate system containing metoprolol tartarate: an in vitro-ex vivo characterization. Curr Drug Deliv. 2009;6(1):113-121.
30. Roblegg E, Frohlich E, Meindl C, Teubl B, Zaversky M, Zimmer A. Evaluation of a physiological in vitro system to study the transport of nanoparticles through the buccal mucosa. Nanotoxicology. 2011. Epub May 18.

31. Fröhlich E, Samberger C, Kueznik T, et al. Cytotoxicity of nanoparticles independent from oxidative stress. J Toxicol Sci. 2009;34(4):363-375.

32. Zhuang J, Ping Q, Song Y, Qi J, Cui Z. Effects of chitosan coating on physical properties and pharmacokinetic behavior of mitoxantrone liposomes. Int J Nanomedicine. 2010;5:407-416.

33. Mertins O, Cardoso MB, Pohlmann AR, da Silveira NP. Structural evaluation of phospholipidic nanovesicles containing small amounts of chitosan. J Nanosci Nanotechnol. 2006;6(8):2425-2431.

34. Mertins O, Schneider PH, Pohlmann AR, da Silveira NP. Interaction between phospholipids bilayer and chitosan in liposomes investigated by 31P NMR spectroscopy. Colloids Surf B Biointerfaces. 2010;75(1):294-299.

35. Henriksen I, Smistad G, Karlsen J. Interactions between liposomes and chitosan. Int J Pharm. 1994;101(3):227-236.

36. Egelhaaf SU, Wehrli E, Mueller M, Adrian M, Schurtenberger P. Determination of the size distribution of lecithin liposomes: a comparative study using freeze fracture, cryoelectron microscopy and dynamic light scattering. J Microsc. 1996;184(3):214-228.

37. Mertins O, Dimova R. Binding of chitosan to phospholipid vesicles studied with isothermal titration calorimetry. Langmuir. 2011;27(9):5506-5515.

38. Ellens H, Bentz J, Szoka FC. pH-induced destabilization of phosphatidylethanolamine-containing liposomes: role of bilayer contact. Biochemistry. 1984;23(7):1532-1538.

39. NayarR, SchroitAJ. Generation of $\mathrm{pH}$-sensitive liposomes: use of large unilamellar vesicles containing N-succinyldioleoylphosphatidylethanolamine. Biochemistry. 1985;24(21):5967-5971.

40. Hassan EE, Gallo JM. A simple rheological method for the in vitro assessment of mucin-polymer bioadhesive bond strength. Pharm Res. 1990;7(5):491-495.

41. He P, Davis SS, Illum L. In vitro evaluation of the mucoadhesive properties of chitosan microspheres. Int J Pharm. 1998;166(1):75-88.

42. Takeuchi H, Yamamoto H, Niwa T, Hino T, Kawashima Y. Enteral absorption of insulin in rats from mucoadhesive chitosan-coated liposomes. Pharm Res. 1996;13(6):896-901.

43. Filipovic-Grcic J, Skalko-Basnet N, Jalsenjak I. Mucoadhesive chitosancoated liposomes: characteristics and stability. J Microencapsul. 2001;18(1):3-12.
International Journal of Nanomedicine

\section{Publish your work in this journal}

The International Journal of Nanomedicine is an international, peerreviewed journal focusing on the application of nanotechnology in diagnostics, therapeutics, and drug delivery systems throughout the biomedical field. This journal is indexed on PubMed Central, MedLine, CAS, SciSearch ${ }^{\circledR}$, Current Contents ${ }^{\circledR} /$ Clinical Medicine,

\section{Dovepress}

Journal Citation Reports/Science Edition, EMBase, Scopus and the Elsevier Bibliographic databases. The manuscript management system is completely online and includes a very quick and fair peer-review system, which is all easy to use. Visit http://www.dovepress.com/ testimonials.php to read real quotes from published authors. 